\title{
The Short-Term Remission of Diabetic Nephropathy After Roux-en-Y Gastric Bypass in Chinese Patients of T2DM with Obesity
}

\author{
Hongwei Zhang ${ }^{1} \cdot$ Jianzhong $\mathrm{Di}^{1} \cdot$ Haoyong $\mathrm{Yu}^{2} \cdot$ Xiaodong $\mathrm{Han}^{1} \cdot \mathrm{Kun}^{1}{ }^{1}$ • \\ Pin Zhang ${ }^{1}$
}

Published online: 30 April 2015

(C) The Author(s) 2015. This article is published with open access at Springerlink.com

\begin{abstract}
The study aimed to examine the effect of gastric bypass on diabetic nephropathy (DN) in Chinese patients. A retrospective study of 101 samples with DN stages I to IV underwent Roux-en-Y gastric bypass. Anthropometric and biochemical parameters were assessed during a 1-year follow-up. Binary logistic regression analysis was used for predictors and ROC for cutoff points. There was significant reduction of medication for diabetes and hypertension. Overall remission of T2DM was $80.2 \%$. Preoperative albumin/ creatinine ratio (ACR) and serum creatinine ( $\mathrm{SCr}$ ) levels could be predictors for DN remission, and the cutoff points were $126 \mathrm{mg} / \mathrm{g}$ cr and $57 \mu \mathrm{mol} / \mathrm{L}$, respectively. Gastric bypass showed improvement of T2DM and DN. Gastric bypass may be more beneficial for those with lower preoperative ACR and SCr.
\end{abstract}

Keywords Gastric bypass · Type 2 diabetes mellitus $\cdot$ Diabetic nephropathy $\cdot$ Microalbuminuria $\cdot$ Albumin/creatinine ratio

The incidence of diabetes mellitus has risen rapidly in the past few decades, and its prevalence has increased from less than $1 \%$ in the Chinese population in the 1980 s [1] to $11.6 \%$ in the

Electronic supplementary material The online version of this article (doi:10.1007/s11695-015-1666-y) contains supplementary material, which is available to authorized users.

Pin Zhang

Pin_Zhang1963@126.com

1 Department of General Surgery, The Sixth People's Hospital Affiliated to Shanghai Jiao Tong University, Shanghai 200233, China

2 Department of Endocrinology and Metabolism, The Sixth People's Hospital Affiliated to Shanghai Jiao Tong University, Shanghai 200233, China
Chinese adult population [2]. Even more serious is that only $25.8 \%$ of these patients received treatment for diabetes, and only $39.7 \%$ of those treated had adequate glycemic control. These findings indicate the importance of diabetes as a public health problem in China with the potential for a major epidemic of diabetes-related complications.

Diabetic nephropathy (DN), also known as diabetic glomerulosclerosis or diabetic kidney disease, is one of the major microvascular complications of type 2 diabetes mellitus (T2DM) [2-4], and is a leading cause of end-stage renal disease (ESRD) with high mortality [5, 6]. Current strategies for the treatment of DN to delay progression involve control of blood glucose and metabolic abnormality [7].

Metabolic surgery has been approved as an effective and potentially useful treatment for T2DM with obesity, especially the Roux-en-Y gastric bypass (RYGB) [8-10]. Several observational studies have shown impressive improvements in glycemic as well as in metabolic disorder, and diabetic complications have shown to be partially reversible due to weight loss and diabetes control [11, 12]. Our prior studies have also shown significant improvement in T2DM including erectile dysfunction and obstructive sleep apnea [13-15].

Though the evidence suggests that RYGB might be a potential treatment for $\mathrm{DN}$, it remains unclear whether the surgery improves progression or provides remission for incipient and overt nephropathy. Therefore, the aim of our study was to investigate whether the RYGB is appropriate for DN.

\section{Methods}

A retrospective study was made between February 2011 and December 2013. A total of 157 patients had RYGB during this 
period, and $101 \mathrm{~T} 2 \mathrm{DM}$ patients with different DN stages were enrolled. The inclusion criteria included (1) age from 18 to 65 years, (2) BMI over $28 \mathrm{~kg} / \mathrm{m}^{2}$, (3) a diagnosis of T2DM based on the criteria of the American Diabetes Association (ADA), and (4) a fasting C-peptide by the oral glucose tolerance test $>1 \mathrm{ng} / \mathrm{mL}$ and a ratio of peak to fasting value $>2 \mathrm{ng} /$ $\mathrm{mL}$. Those with established diagnoses of type 1 diabetes, latent autoimmune diabetes in adults, malignancy, debilitating disease, unresolved psychiatric illness, or substance abuse were excluded from the study.

$\mathrm{DN}$ is divided into five stages according to the Morgenson standard [16]: (I) hyperfunction with raised glomerular filtration rate (GFR) and increased kidney volume, (II) normoalbuminuria with the thickening of the glomerular basement membrane (GBM) and expansion of the mesangial matrix, (III) incipient DN with microalbuminuria (MAU) 30$299 \mathrm{mg} /$ day, (IV) overt DN with MAU >300 mg/day, and (V) uremia with ESRD. Patients with DN stage V were excluded from this study for surgical contraindication. Remission in DN stages III and IV was defined as MAU $<30 \mathrm{mg} /$ day. This study focused on patients in DN stage III and IV, which are more likely to develop end-stage renal disease.

Medical history, current medications, anthropometric evaluations including weight, BMI, percent of excess weight loss (\%EWL), waistline, and hipline were recorded before and after bypass surgery. Biochemical parameters were evaluated by obtaining blood samples after an overnight fast and included fasting glucose, postprandial glucose followed by OGTT, fasting C-peptide, glycated hemoglobin ( $\mathrm{HbA1c}$ ), glycosylated serum protein (GSP), lipid profile (cholesterol, triglyceride, high-density, and low-density lipoprotein), and renal function profile (blood urea nitrogen [BUN], serum creatinine [SCr], and uric acid [UA]). In addition, the screening of DN with GFR, urine albumin/creatinine ratio (ACR), and 24-h microalbuminuria were evaluated. Insulin resistance level and $\beta$-cell function were measured by the homeostasis model assessment insulin resistance (HOMA-IR) using the formula $\mathrm{mIU} / \mathrm{mmol} / \mathrm{L} 2=$ fasting insulin $(\mathrm{mIU} / \mathrm{L}) \times$ fasting glucose $(\mathrm{mmol} / \mathrm{L}) / 22.5$ and the homeostasis model assessment $\beta$ cell (HOMA- $\beta$ ) using $\%=20 \times$ fasting insulin $(\mathrm{mIU} / \mathrm{L}) /$ fasting glucose $(\mathrm{mmol} / \mathrm{L})-3.5)(\%)$.

Remission of T2DM was defined as an HbA1c level $<6.5 \%$ and a fasting glucose concentration $<7.0 \mathrm{mmol} / \mathrm{L}$ for 1 year or more without active pharmacologic intervention [17]. Plasma glucose concentrations were measured using the glucose oxidase method. Serum insulin and C-peptide levels were quantified using radioimmunoassays with specific insulin and C-peptide detection kits according to the manufacturer's instructions (Beijing North Institute of Biological Technology, Beijing, China). HbA1c was measured by HPLC (Menarini, former reference range 4.0-6.0\%). GSP was measured by ELISA (Hitachi 7100, Tokyo, Japan). The serum lipid and renal function profile were measured using standard commercial methods on a parallel, multichannel analyzer (Hitachi 7600-020, Tokyo, Japan). ACR and 24-h MAU were measured by chemiluminescence immunoassay (Siemens, DCA Vantage, Germany), and GFR was measured by $99 \mathrm{~m}$ Tc-DTPA (Siemens, SPECT, E CAM Single, Germany).

Of the 101 patients, $45(44.6 \%)$ were female. The mean age of all participants was $47.6 \pm 11.9$ years, and the mean duration of T2DM was $7.63 \pm 4.84$ years. Hypertension was diagnosed in 40 (39.6 \%). All patients were divided into four groups according to their DN stage diagnosis.

Nearly $50 \%$ were treated with insulin and more than $60 \%$ with oral diabetes medications in each group preoperatively. Patients in DN stage I with hypertension (25.8 \%) had the least proportion of antihypertensives (12.9\%). Most of the antihypertensives taken were angiotensin-converting enzyme
Table 1 Characteristics of the participants

\begin{tabular}{lllll}
\hline & DN1 & DN2 & DN3 & DN4 \\
\hline Gender (F/M) & $16: 15$ & $08: 26$ & $11: 10$ & $10: 05$ \\
Age (years) & $41.35 \pm 9.37$ & $54.91 \pm 10.23$ & $47.62 \pm 13.70$ & $44.13 \pm 8.70$ \\
Duration (years) & $4.87 \pm 2.85$ & $9.94 \pm 4.57$ & $7.18 \pm 5.60$ & $8.33 \pm 4.92$ \\
Hypertension & $8(25.8 \%)$ & $14(41.2 \%)$ & $11(52.4 \%)$ & $7(46.7 \%)$ \\
Medication use before surgery & & & & \\
Insulin & $10(47.6 \%)$ & $17(50.0 \%)$ & $9(42.9 \%)$ & $13(46.7 \%)$ \\
Oral diabetes medications & $19(61.3 \%)$ & $25(73.5 \%)$ & $13(61.9 \%)$ & $9(60.0 \%)$ \\
Antihypertensives & $4(12.9 \%)$ & $12(35.3 \%)$ & $9(42.9 \%)$ & $6(40.0 \%)$ \\
Antihyperlipidemia & $15(48.4 \%)$ & $12(35.3 \%)$ & $13(61.9 \%)$ & $8(53.3 \%)$ \\
Medication use 1 year after surgery $\%)$ & & $1(6.7 \%)$ \\
Insulin & $1(3.2 \%)$ & $2(5.9 \%)$ & $1(4.8 \%)$ & $1(6.7 \%)$ \\
Oral diabetes medications & $2(6.5 \%)$ & $2(5.9 \%)$ & $2(9.5 \%)$ & $1(6.7 \%)$ \\
Antihypertensives & $2(6.5 \%)$ & $5(14.7 \%)$ & $2(9.5 \%)$ & 0 \\
Antihyperlipidemia & $1(3.2 \%)$ & $1(2.9 \%)$ & $1(4.8 \%)$ & \\
\hline
\end{tabular}


inhibitor (ACEI) or/with angiotensin receptor blocker (ARB). Antihyperlipidemia medication was taken by 61.9 and $60 \%$ separately in DN stages III and IV while in DN stages I and II, it was 48.4 and $35.3 \%$, respectively (Table 1 ).

\section{Surgical Technique}

All patients had laparoscopic Roux-en-Y gastric bypass (LRYGB) by the same surgical team with over 50 cases of experience. The patient was placed in reverse Trendelenburg position and the surgeon positioned between the patient's legs. Five trocars are inserted under direct laparoscopic vision. A gastric pouch of approximately $30 \mathrm{~mL}$ is created with mechanical sutures preventing the transection of the left gastric artery. The angle of Treitz is then identified, and a 100-cm-long jejunal loop (biliopancreatic limb) is ascended anterior to the colon and anastomosed to the gastric pouch with mechanical linear suture $(30 \mathrm{~mm})$. A lateral jejuno-jejunal anastomosis adjacent to gastro-jejunal anastomosis is made $100 \mathrm{~cm}$ away from the previous nastomosis (alimentary limb) with a mechanical linear stapler.

\section{Statistics Analysis}

All statistics were calculated with SPSS statistical software (version 20.0; SPSS Inc., Armonk, NY, USA). Paired $t$ tests were used to compare variables before and after the study. Contingency tables of categorical variables were analyzed by Fisher's exact test (grade and gender in remission of DN stages III and IV). Correlations between clinical parameters and MAU were analyzed by partial correlation analysis. Multiple stepwise logistic regression analysis was performed to assess the independent predictive effects of the variables on remission of DN. The measure index was set as blood pressure, albumin/creatinine ratio and serum creatinine, and the remission standard as MAU $<30 \mathrm{mg}$ /day. The predictors' cutoff point was evaluated by Youden's Index with the receiver operating characteristic (ROC) curve. Data are presented as the mean $\pm \mathrm{SD}$. Statistical significance was defined as $P<0.05$.

\section{Results}

Four groups are comparable in BMI $(F=1.529, P=0.212)$, patients in DN stage II were older $(F=0.915, P=0.000)$ and longer T2DM duration $(F=6.975, P=0.000)$. One year after surgery, there was a significant reduction of each type of medication used in each group (Table 1).

The overall remission of T2DM was $80.20 \%$, and in DN stage I, it was $95.2 \%$, DN stage II was $86.7 \%$, DN stage III was $23.8 \%$, and DN stage IV was $46.7 \%$. Changes in anthropometric and biochemical parameters of all patients before and after surgery are shown in Table 2.
The overall remission of DN stages III and IV was $58.3 \%$. There was no significant difference in age or BMI between two groups. Analysis showed that the remission parameter microalbuminuria was correlated with albumin/creatinine ratio, systolic blood pressure (SBP), serum creatinine, HomaIR, Homa- $\beta$, fasting C-peptide, and cholesterol levels (Table 3). According to the DN remission standard for incipient (stage III) and overt (stage IV) nephropathy, the preoperative clinical variables related to diabetes were assessed for their predictive values (Table 4). Analysis showed that in DN patients with stage III, there were lower microalbuminuria, albumin/creatinine ratio, systolic blood pressure, blood urea nitrogen, and serum creatinine levels compared with those without remission. Preoperative BMI, diabetes duration, waistline, and GFR had no significant differences between the two groups. The results of multivariate regression analysis confirmed that systolic blood pressure, serum creatinine and albumin/creatinine ratio were clinical predictors for $\mathrm{DN}$ remission (Table 5). Further analysis for predicting the DN remission using ROC showed that AUC in serum creatinine and albumin/creatinine ratio was 0.770 and 0.917 with the cutoff point of $57 \mu \mathrm{mol} / \mathrm{L}$ in serum creatinine and $126(\mathrm{mg} / \mathrm{g} \mathrm{cr})$ in albumin/creatinine ratio (Table 6).

\section{Discussion}

Recently, there have been many encouraging reports suggesting that gastric bypass is an effective, stand-alone procedure for T2DM with obesity. However, before discussing DN remission, the primary concern should be about safety. In our study, all surgeries were performed by one team with experience of over 50 cases of LRYGB. An important aspect in the learning curve for successful metabolic surgery, according to Huang [18], is having experience of approximately 30 cases. Common complications such as fistula, obstruction, stenosis, and hemorrhage did not occur in our study. In addition, there was no acute renal failure in the patients with DN stage III or IV.

The primary aim of DN treatment is currently focused on delaying its progression by improving the control of T2DM and of hemodynamic abnormalities [8]. Evidence shows that $\mathrm{DN}$ is characterized by structural and functional changes, which show an imbalance in afferent and efferent arteriolar resistance and may result in increased glomerular hydrostatic pressure and hyperfiltration $[19,20]$.

The present study advocates that vascular endothelial dysfunction leads to changes in the renal vascular system and contributes to the development of DN. Good glycemic control is effective in reducing diabetic microvascular complication [21]. Hemodynamic dysfunction could be another factor resulting in DN, and diabetic endothelial nitric oxide synthase 


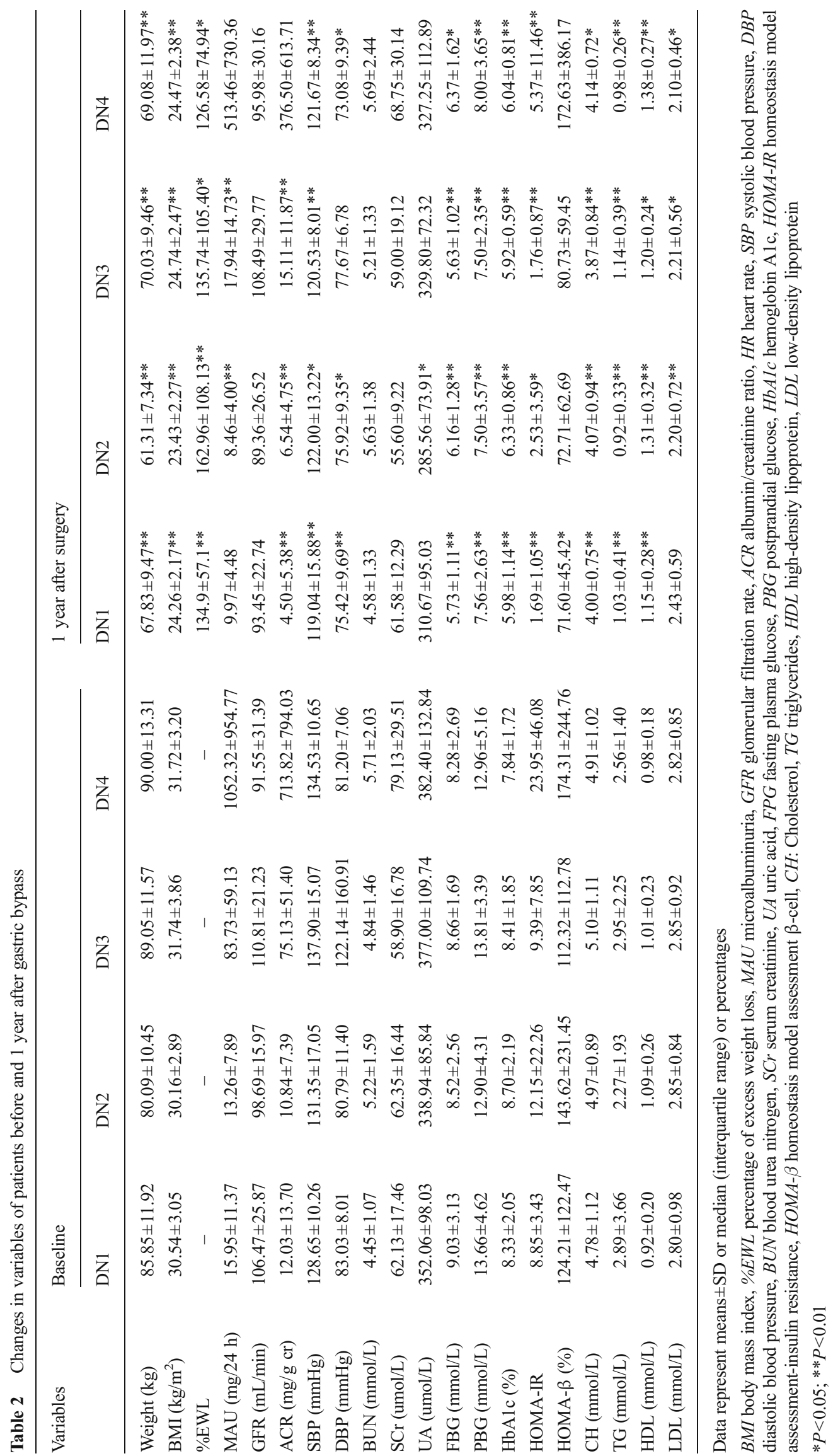


Table 3 The correlation test for MAU in patients with DN III/IV

\begin{tabular}{|c|c|c|}
\hline & \multicolumn{2}{|l|}{ MAU } \\
\hline & $r$ & $P$ \\
\hline Age (years) & -0.063 & 0.543 \\
\hline Duration (years) & 0.099 & 0.346 \\
\hline GFR (mL/min) & -0.161 & 0.062 \\
\hline ACR (mg/g cr) & 0.962 & $0.000 * *$ \\
\hline Weight (kg) & 0.040 & 0.479 \\
\hline BMI $\left(\mathrm{kg} / \mathrm{m}^{2}\right)$ & 0.105 & 0.065 \\
\hline$\%$ EWL & -0.003 & 0.961 \\
\hline Waistline $(\mathrm{cm})$ & 0.041 & 0.474 \\
\hline Hipline (cm) & 0.015 & 0.794 \\
\hline HR (bpm) & 0.069 & 0.229 \\
\hline SBP (mmHg) & 0.126 & $0.026^{*}$ \\
\hline DBP (mmHg) & -0.005 & 0.936 \\
\hline BUN (mmol/L) & 0.110 & 0.053 \\
\hline $\mathrm{SCr}(\mu \mathrm{mol} / \mathrm{L})$ & 0.256 & $0.000 * *$ \\
\hline $\mathrm{UA}(\mu \mathrm{mol} / \mathrm{L})$ & 0.067 & 0.242 \\
\hline FBG $(\mathrm{mmol} / \mathrm{L})$ & 0.072 & 0.207 \\
\hline PBG (mmol/L) & 0.058 & 0.310 \\
\hline HbA1c (\%) & 0.049 & 0.310 \\
\hline GSP (\%) & -0.069 & 0.226 \\
\hline HOMA-IR & 0.336 & $0.000^{* *}$ \\
\hline HOMA- $\beta(\%)$ & 0.264 & $0.000 * *$ \\
\hline CP 0 (ng/mL) & 0.112 & $0.049 *$ \\
\hline $\mathrm{CH}(\mathrm{mmol} / \mathrm{L})$ & 0.173 & $0.002 * *$ \\
\hline TG (mmol/L) & 0.051 & 0.370 \\
\hline HDL (mmol/L) & 0.083 & 0.143 \\
\hline $\mathrm{LDL}(\mathrm{mmol} / \mathrm{L})$ & 0.083 & 0.143 \\
\hline
\end{tabular}

$B M I$ body mass index, $\% E W L$ percentage of excess weight loss, $M A U$ microalbuminuria, GFR glomerular filtration rate, $A C R$ albumin/creatinine ratio, $H R$ heart rate, $S B P$ systolic blood pressure, $D B P$ diastolic blood pressure, $B U N$ blood urea nitrogen, $S C r$ serum creatinine, $U A$ uric acid, $F P G$ fasting plasma glucose, $P B G$ postprandial glucose, $H b A l c$ hemoglobin A1c, GSP glycosylated serum protein, $C P O$ fasting C-peptide, HOMA-IR homeostasis model assessment-insulin resistance, HOMA- $\beta$ homeostasis model assessment $\beta$-cell, $T G$ triglycerides, $\mathrm{CH}$ cholesterol, $H D L$ high-density lipoprotein, $L D L$ low-density lipoprotein

knockout mice develop more severe glomerular lesions and proteinuria compared to wild-type mice [22].

A recently described mechanism of proximal tubule injury that involved the formation of advanced glycation endproducts (AGE) by nonenzymatic binding of glucose to proteins, lipids, and nucleic acids can lead to alteration of protein structure and function, oxidative stress, and expression of proinflammatory cytokines and growth factors [23], which cause the development of DN [24]. In addition, evidence suggests that genetic predisposition, hyperglycemia, hypertension, and dyslipidemia, all factors associated to metabolic syndrome, contribute to the induction and progression of DN [25]. Thus,
Table 4 Comparison of preoperative factors of DN remission in grade III and IV

\begin{tabular}{|c|c|c|c|}
\hline Factor & Remission & No remission & $P$ value \\
\hline DN grade (III/IV) & $19: 2$ & $2: 13$ & $0.000^{* *}$ \\
\hline Gender (F/M) & $11: 10$ & $4: 11$ & 0.123 \\
\hline Age (years) & $46.48 \pm 13.60$ & $45.73 \pm 9.31$ & 0.856 \\
\hline Duration (years) & $6.85 \pm 5.58$ & $8.80 \pm 4.78$ & 0.280 \\
\hline Weight (kg) & $88.79 \pm 11.72$ & $90.37 \pm 13.09$ & 0.706 \\
\hline BMI $\left(\mathrm{kg} / \mathrm{m}^{2}\right)$ & $31.94 \pm 3.86$ & $31.44 \pm 3.17$ & 0.682 \\
\hline Waistline (cm) & $106.14 \pm 9.96$ & $104.53 \pm 8.69$ & 0.618 \\
\hline Hipline (cm) & $109.05 \pm 7.43$ & $107.00 \pm 6.29$ & 0.392 \\
\hline MAU (mg/24 h) & $132.32 \pm 201.92$ & $984.30 \pm 995.50$ & $0.005 * *$ \\
\hline GFR (mL/min) & $109.89 \pm 24.56$ & $92.78 \pm 28.73$ & 0.067 \\
\hline $\mathrm{ACR}(\mathrm{mg} / \mathrm{g} \mathrm{cr})$ & $120.37 \pm 169.61$ & $650.48 \pm 820.04$ & $0.026^{*}$ \\
\hline HR (bpm) & $77.00 \pm 6.62$ & $79.27 \pm 3.41$ & 0.234 \\
\hline SBP (mmHg) & $129.28 \pm 15.35$ & $138 \pm 9.82$ & $0.049^{*}$ \\
\hline DBP (mmHg) & $122.14 \pm 160.91$ & $81.20 \pm 7.06$ & 0.334 \\
\hline BUN (mmol/L) & $4.72 \pm 1.45$ & $5.87 \pm 1.96$ & $0.049^{*}$ \\
\hline $\mathrm{SCr}(\mu \mathrm{mol} / \mathrm{L})$ & $57.62 \pm 16.11$ & $80.93 \pm 28.61$ & $0.010 * *$ \\
\hline $\mathrm{UA}(\mu \mathrm{mol} / \mathrm{L})$ & $372.00 \pm 110.74$ & $389.40 \pm 131.00$ & 0.669 \\
\hline FBG (mmol/L) & $8.67 \pm 1.67$ & $8.27 \pm 2.70$ & 0.585 \\
\hline PBG (mmol/L) & $13.78 \pm 3.46$ & $13.00 \pm 5.11$ & 0.586 \\
\hline CP 0 (ng/mL) & $2.73 \pm 1.25$ & $3.04 \pm 1.86$ & 0.550 \\
\hline HbAlc (\%) & $8.27 \pm 1.83$ & $8.05 \pm 1.79$ & 0.722 \\
\hline GSP (\%) & $19.45 \pm 4.86$ & $17.97 \pm 4.00$ & 0.353 \\
\hline HOMA-IR & $8.81 \pm 7.30$ & $24.76 \pm 45.92$ & 0.125 \\
\hline HOMA- $\beta(\%)$ & $105.93 \pm 108.72$ & $1042.83 \pm 3335.05$ & 0.204 \\
\hline $\mathrm{CH}(\mathrm{mmol} / \mathrm{L})$ & $5.11 \pm 1.13$ & $4.90 \pm 0.99$ & 0.574 \\
\hline $\mathrm{TG}(\mathrm{mmol} / \mathrm{L})$ & $2.85 \pm 2.28$ & $2.71 \pm 1.36$ & 0.842 \\
\hline HDL (mmol/L) & $1.02 \pm 0.23$ & $0.95 \pm 0.20$ & 0.339 \\
\hline LDL (mmol/L) & $2.85 \pm 0.92$ & $2.82 \pm 0.85$ & 0.923 \\
\hline
\end{tabular}

$B M I$ body mass index, $M A U$ microalbuminuria, GFR glomerular filtration rate, $A C R$ albumin/creatinine ratio, $H R$ heart rate, $S B P$ systolic blood pressure, $D B P$ diastolic blood pressure, $B U N$ blood urea nitrogen, $S C r$ serum creatinine, $U A$ uric acid, $F P G$ fasting plasma glucose, $P B G$ postprandial glucose, $H b A 1 c$ hemoglobin A1c, GSP glycosylated serum protein, $C P O$ fasting C-peptide, HOMA-IR homeostasis model assessmentinsulin resistance, HOMA- $\beta$ homeostasis model assessment $\beta$-cell, TG triglycerides, $C H$ cholesterol, $H D L$ high-density lipoprotein, $L D L$ lowdensity lipoprotein

Data represent means $\pm \mathrm{SD}$ or median (interquartile range) or percentages ${ }^{*} P<0.05$; ** $P<0.01$

controlling and improving metabolic syndrome could be the key link to DN treatment. However, current strategies including diabetic medicines, antihypertensives, and antilipid medicine are not effective.

This study confirms the remarkable efficacy of gastric bypass for treatment of T2DM with obesity. The percentage of female patients participating was $44.6 \%$, which is lower than in other studies. The overall remission of T2DM after 1 year was $80.20 \%$. The central obesity status, hypertension, and 
Table 5 Multiple logistic regression analysis of variables at baseline

\begin{tabular}{lllll}
\hline & $B$ & SE & $P$ value & Exp(B) \\
\hline SBP & -0.208 & 0.087 & 0.017 & 0.812 \\
SCr & 0.093 & 0.044 & 0.034 & 1.098 \\
ACR & 0.005 & 0.002 & 0.019 & 1.005 \\
\hline
\end{tabular}

Predicted Probability is of membership for none remission

$B$ coefficient of regression, $S E$ standard error, $\operatorname{Exp}(\mathrm{B})$ odd ration, $S B P$ systolic blood pressure, $S C r$ serum creatinine, $A C R$ albumin/creatinine ratio

lipid levels were significantly improved in all DN groups. Further analysis showed that patients in DN stage I were younger with shorter diabetes duration, less insulin resistance, and better blood pressure control compared with those in DN stages III and IV before surgery. Despite the differences in the four groups, the 1-year follow-up showed that gastric bypass can also lead to similar recovery of metabolic disorder. Although HOMA-IR and HOMA- $\beta$ in DN stage IV showed impressive improvement, they were significantly higher than in the other three groups 1 year following surgery, which represents greater insulin resistance and poor $\beta$-cell function indicating progression of DN. Furthermore, the medication used for diabetes, hypertension, and hyperlipidemia was reduced significantly in all groups after surgery.

Most patients with hypertension in our study used ACEI and/or ARB, which could reduce the risk of albuminuria to control blood pressure [26]. After surgery, antihypertensive medication was significantly reduced and most patients became well controlled with blood pressure under 130/ $80 \mathrm{mmHg}$. A study by Pohl et al. [27] indicated that a target systolic blood pressure of $120-130 \mathrm{mmHg}$ provided tremendous benefit to renal outcomes. Our study found that patients in DN stages I to IV maintained continuous improvement for metabolic disorder in 1-year short term. In addition, weight loss and better diabetic control improved the lifestyle of patients and also provided further benefit by slowing down the progression of DN.

The renal function index including serum creatinine, blood urea nitrogen, uric acid, and GFR had no significant changes.

Table 6 ROC for remission of DN in predictors

\begin{tabular}{llllll}
\hline & AUC & $p$ valve & $95 \%$ CI & & Cutoff point \\
\hline SBP & 0.4 & 0.312 & 0.212 & 0.588 & - \\
SCr & 0.77 & 0.006 & 0.616 & 0.923 & 57 \\
ACR & 0.917 & 0.000 & 0.826 & 1.000 & 126 \\
\hline
\end{tabular}

SBP had no predictive value $(\mathrm{AUC}<0.5)$. For $\mathrm{SCr}(\mathrm{AUC}=0.77)$ and ACR (AUC=0.917), ACR had best predictive value and accuracy for predict $\mathrm{DN}$ remission, $\mathrm{AUC}>0.9$

$S B P$ systolic blood pressure, $S C r$ serum creatinine, $A C R$ albumin/creatinine ratio, $A U C$ area under curve
However, microalbuminuria and albumin/creatinine ratio were significantly reduced in DN stages I, II, and III, but not in patients in stage IV. The mean level reduced continuously. The microalbuminuria reflects the glomerular filtration function due to the change in endothelial cell permeability and glomerular basement membrane. The GFR is affected by several factors including the glomerular filtration function, glomerular hydrostatic pressure, and colloid osmotic pressure. Therefore, we speculate that gastric bypass could improve the capillary membrane permeability, but the complex connection between capillary hemodynamics and gastric bypass still needs further research.

Patients with DN at the time of T2DM diagnosis can go unrecognized for years. Albuminuria is common among patients with established diabetes, and becomes more prevalent with worsening glucose tolerance [28]. DN stages III and IV, which are also called incipient and overt nephropathy, are more likely to develop end-stage renal disease [29]. Therefore, our study has focused on the change of microalbuminuria especially in DN stages III and IV following surgery. The remission of DN (decrease in MAU to $<30 \mathrm{mg} / 24 \mathrm{~h}$ ) could slow the progression of nephropathy and may prevent the development of end-stage renal disease. As for patients in stages I and II, DN were controlled by reducing of GFR and microalbuminuria 1 year after surgery.

Our study found that patients in DN stage III are more likely to get remission compared with stage IV, but gender, age, and diabetic duration were not correlated. The renal function index including microalbuminuria, albumin/creatinine ratio, serum creatinine, and blood urea nitrogen had a significant difference in both patients with and without remission before surgery. Further analysis shows significant correlation with albumin/creatinine ratio, serum creatinine, systolic blood pressure, cholesterol and insulin sensitivity, and insulin resistance. This result confirms the relationship between DN and metabolic disorder.

In our study, patients with increased baseline of systolic blood pressure, serum creatinine, or albumin/creatinine ratio are less likely to achieve remission of DN, and the ROC curve revealed that serum creatinine and albumin/creatinine ratio are potential predicators for remission failure of incipient and overt DN. Youden's Index showed that the cutoff point for serum creatinine and albumin/creatinine ratio were $57 \mu \mathrm{mol} /$ $\mathrm{L}$ and $126 \mathrm{mg} / \mathrm{g} \mathrm{cr}$, respectively. As a normal value of the serum creatinine cutoff point with less applicability, the albumin/creatinine ratio cutoff point may have greater clinical value. Although most patients in stage IV did not reach the remission standard, the reduction of microalbuminuria helped to stabilize DN.

Our study has several limitations such as retrospective design, small population size, and the limited follow-up duration which may have interfered with the results. The study by Alexander et al. showed that loss of excess body weight had 
the most positive effect in patients with obesity-related focal segmental glomerulosclerosis [30], which differs from our study. Therefore, a study with a long-term follow-up and a large population sample is necessary to strengthen and establish the cutoff values for serum creatinine and albumin/ creatinine ratio. In addition, a further study with prospective design is required to confirm whether DN remission after RYGB is a temporary result or has a long-term effect. It is also necessary to perform a pathology and pathophysiology study of DN before and after surgery.

In conclusion, the beneficial results observed in our study demonstrate that gastric bypass should be considered in Chinese patients with T2DM and obesity. The RYGB shows the possibility of slowing down progression of diabetic nephropathy. Furthermore, albumin/creatinine ratio could help predict the remission of $\mathrm{DN}$.

Conflict of Interest The authors have no commercial associations that might be a conflict of interest in relation to this article.

Statement of Informed Consent Informed consent was obtained from all individual participants included in the study.

Statement of Human and Animal Rights This study was approved by the Ethics Committee of the Shanghai Jiao Tong University Affiliated Sixth People's Hospital and complied with the Declaration of Helsinki.

Open Access This article is distributed under the terms of the Creative Commons Attribution 4.0 International License (http:// creativecommons.org/licenses/by/4.0/), which permits unrestricted use, distribution, and reproduction in any medium, provided you give appropriate credit to the original author(s) and the source, provide a link to the Creative Commons license, and indicate if changes were made.

\section{References}

1. National Diabetes Research Group. A mass survey of diabetes mellitus in a pospulation of 300,000 in 14 provinces and municipalities in China. Zhonghua Nei Ke Za Zhi. 1981;20(11): 678-83.

2. Xu Y, Wang L, He J, et al. Prevalence and control of diabetes in Chinese adults. JAMA. 2013;310(9):948-59.

3. Kikkawa R, Koya D, Haneda M. Progression of diabetic nephropathy. Am J Kidney Dis. 2003;41:S19-21.

4. Atkins RC, Zimmet P. Diabetic kidney disease: act now or pay later. Kidney Int. 2010;77:375-7.

5. Lim AKH. Diabetic nephropathy - complications and treatment. Int J Nephrol Renovasc Dis. 2014;7:361-81.

6. International Diabetes Federation. IDF Diabetes Atlas 6thed Brussels, Belgium: International Diabetes Federation; 2013. Available from: http://www.idf.org/sites/default/files/EN_6E_ Atlas_Full_0.pdf. Accessed September 2, 2014.2.

7. Lv M, Chen Z, Hu GY, et al. Therapeutic strategies of diabetic nephropathy: recent progress and future perspectives. Drug Discov Today. 2014;00:1-15.

8. Dixon JB, Zimmet P, Alberti KG, et al. Bariatric surgery: an IDF statement for obese type 2 diabetes. Diabet Med. 2011;28:628-42.
9. Mingrone G, Panunzi S, De Gaetano A, et al. Bariatric surgery versus conventional medical therapy for type 2 diabetes. $\mathrm{N}$ Engl J Med. 2012;366(17):1577-85.

10. Schauer PR, Bhatt DL, Kirwan JP, et al. Bariatric surgery versus intensive medical therapy for diabetes - 3-year outcomes. N Engl J Med. 2014;370(21):2002-13.

11. Buchwald H, Estok R, Fahrbach K, et al. Weight and type 2 diabetes after bariatric surgery: systematic review and meta-analysis. Am J Med. 2009;122(3):248-56.

12. Vetter ML, Cardillo S, Rickels MR, et al. Narrative review: effect of bariatric surgery on type 2 diabetes mellitus. Ann Intern Med. 2009;150:94-103. 480.

13. Yu H, Di J, Bao Y, et al. Visceral fat area as a new predictor of shortterm diabetes remission after Roux-en-Y gastric bypass surgery in Chinese patients with a body mass index less than $35 \mathrm{~kg} / \mathrm{m}^{2}$. Surg Obes Relat Dis. 2014.

14. Kun L, Pin Z, Jianzhong D, et al. Significant improvement of erectile function after Roux-en-Y gastric bypass surgery in obese Chinese men with erectile dysfunction. Obes Surg. 2014. [Epub ahead of print].

15. Zou J, Zhang P, Yu H, et al. Effect of laparoscopic Roux-en-Y gastric bypass surgery on obstructive sleep apnea in a Chinese population with obesity and T2DM. Obes Surg. 2014. [Epub ahead of print].

16. Mogensen CE, Schmitz O. The diabetic kidney: from hyperfiltration and microalbuminuria to end-stage renal failure. Med Clin N Am. 1988;72(6):1465-92.

17. Buse JB, Caprio S, Cefalu WT, et al. How do we define cure of diabetes. Diabetes Care. 2009;32:2133-5.

18. Huang CK, Lee YC, Hung CM, et al. Laparoscopic Rouxen-Y gastric bypass for morbidly obese Chinese patients: learning curve, advocacy and complications. Obes Surg. 2008;18(7):776-81.

19. Huang W, Gallois Y, Bouby N, et al. Genetically increased angiotensin I-converting enzyme level and renal complications in the diabetic mouse. Proc Natl Acad Sci U S A. 2001;98(23): $13330-4$.

20. Rudberg S, Rasmussen LM, Bangstad HJ, et al. Influence of insertion/ deletion polymorphism in the ACE-I gene on the progression of diabetic glomerulopathy in type 1 diabetic patients with microalbuminuria. Diabetes Care. 2000;23(4):544-8.

21. Rossing P, Hougaard P, Parving HH. Risk factors for development of incipient and overt diabetic nephropathy in type 1 diabetic patients: a 10-year prospective observational study. Diabetes Care. 2002;25(5):859-64.

22. Kanetsuna Y, Takahashi K, Nagata M, et al. Deficiency of endothelial nitric-oxide synthase confers susceptibility to diabetic nephropathy in nephropathy-resistant inbred mice. Am J Pathol. 2007;170(5):1473-84.

23. Lanaspa MA, Ishimoto T, Cicerchi C, et al. Endogenous fructose production and fructokinase activation mediate renal injury in diabetic nephropathy. J Am Soc Nephrol. 2014.

24. Sheetz MJ, King GL. Molecular understanding of hyperglycemia's adverse effects for diabetic complications. JAMA. 2002;288(20): 2579-88.

25. Mooyaart AL. Genetic associations in diabetic nephropathy. Clin Exp Nephrol. 2013;54:544-53.

26. Viberti G, Mogensen CE, Groop LC, et al. European Microalbuminuria Captopril Study Group. Effect of captopril on progression to clinical proteinuria in patients with insulindependent diabetes mellitus and microalbuminuria. JAMA. 1994;271(4):275-9.

27. Pohl MA, Blumenthal S, Cordonnier DJ, et al. Independent and additive impact of blood pressure control and angiotensin II receptor blockade on renal outcomes in the irbesartan diabetic 
nephropathy trial: clinical implications and limitations. J Am Soc Nephrol. 2005;16:3027-37.

28. Tapp RJ, Shaw JE, Zimmet PZ, et al. Albuminuria is evident in the early stages of diabetes onset: results from the Australian Diabetes, Obesity, and Lifestyle Study (AusDiab). Am J Kidney Dis. 2004;44(5):792-8.
29. Molitch ME, DeFronzo RA, Franz MJ, et al. American Diabetes Association. Nephropathy in diabetes. Diabetes Care. 2004;27 Suppl 1:S79-83.

30. Alexander JW, Goodman HR, Hawver LM, et al. Improvement and stabilization of chronic kidney disease after gastric bypass. Surg Obes Relat Dis. 2009;2:237-41. 